\title{
Aprendizaje-servicio al servicio de la Comunidad Educadora
}

\author{
Patrizia Lotti \\ Istituto Nazionale Documentazione Innovazione Ricerca Educativa, Italia \\ Francesca Betti \\ FILCAMS CGIL, Italia
}

\section{Resumen}

La evolución tecnológica, productiva y organizativa determina transformaciónes trascendentales en el mundo del trabajo que requieren un cambio sustancial y adecuado del sistema educativo. La profunda inseguridad y sensación de precariedad que sienten las personas está acentuada por la única visión económica que en las últimas décadas ha determinado la afirmación del individualismo solipsista. La gestión de estas transformaciónes trascendentales solicita competencias de tipo colaborativo y solidario. El aprendizaje-servicio puede permitir llegar al núcleo del problema y a traves de un intercambio ventajoso entre conocimientos interdiciplinarios y competencias experimentales, volver a motivar la relación educativa en sentido participativo; unido, por lo tanto, con un desarrollo democrático. Para que esto suceda es necesaria una espontánea difusión de las prácticas, que vaya más allá de la moda y que permita incidir en la inercia de una realidad que se ha adaptado burocráticamente a las contínuas novedades de las normas. Se propone el crecimiento de un movimiento que difunda desde abajo, tal vez ayudado de un intercambio reticular de experiencias y conocimientos que permita una nueva centralidad política de un sistema educativo proactivo.

\section{Palabras clave}

Aprendizaje-servicio, institucionalización, competencias.

Fecha de recepción: 18/III/2019

Fecha de aceptación: 10/V/2019 


\title{
Service-learning at the service of the education community
}

\begin{abstract}
Technological, productive and organizational advancements trigger transcendental transformations in the workplace, which necessarily require a substantial and suitable change in the educational system. Managing these transformations require collaborative and solidary competences. Furthermore, new economic approaches linked to the development of capabilities and character open up an educational system based on the learning of care, responsibility and solidarity. Service-learning can make it possible to reach the core of the issue and, through a valuable exchange of interdisciplinary knowledge and competences; it can also re-ignite educational participatory practices, which are linked to democratic development. For this to happen, it is important to disseminate service-learning practices widely, going beyond its fashionable acceptance and combating the inertia of a reality that has bureaucratically conformed to the norm even with continuous novel developments, whilst also enabling the growth of a movement that has been developing from the bottom up, with the help of a reticular exchange of experiences and knowledge allowing for a new political centrality of an educational system that is finally proactive.
\end{abstract}

\section{Keywords}

Service-learning, institutionalization, competencies. 


\section{Relación y cuidado como antídoto a la desilusión en la vida, el trabajo y la escuela}

\author{
"Los seres humanos prefieren pensar \\ en términos de historias en lugar de \\ hechos, números o ecuaciones, $y$ \\ cuanto más simple, mejor" (Harari, \\ 2018 , p. 19). Durante el siglo XX, las \\ elites globales contaron el pasado y \\ predijeron el futuro a través de tres \\ grandes narrativas: la fascista, la \\ comunista y la liberal. Esta última se \\ desarrolló y se extendió a nivel mundial \\ a finales del siglo $X X$ e inicios del siglo \\ XXI, y luego comenzó a declinar a \\ partir de la crisis financiera de 2008, \\ dando lugar a nuevas formas de \\ democracia iliberal, que en algunos \\ casos degeneraron en regímenes \\ dictatoriales reales. "Esto es lo que \\ probablemente está sucediendo en todo \\ el mundo, ya que el vacío dejado por la \\ crisis del liberalismo se llena \\ temporalmente con fantasías \\ nostálgicas del glorioso pasado local" \\ (Harari, 2018, p. 37).
}

Cada narración, incluso cuando no proporciona respuestas a los problemas graves y contingentes que enfrenta, como el liberal, es tranquilizadora y clarificadora y al ser privado de repente, impresiona, desorienta, haciendo perder puntos de referencia para interpretar la realidad. Hoy en día, la raza humana se encuentra en una fase de desilusión nihilista, ha perdido la fe en las narrativas antiguas, pero aún no ha abrazado otras nuevas (Harari, 2018, p. 40): las personas no solo están aniquiladas, sino también

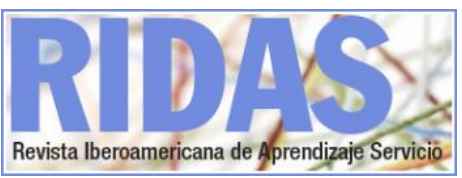

"reconstruidas". Las "revoluciones gemelas" generadas, por un lado, por las tecnologías informáticas, por el otro, por las biológicas, de hecho "podrían reestructurar no solo las economías y las sociedades, sino también nuestros cuerpos y nuestras mentes", de hecho ya han inaugurado una "mutación antropológica sin precedentes". En una época en constante cambio, donde la estabilidad y la seguridad se han perdido de vista, donde ya no existe un mundo común, asistimos al "eclipse de una dimensión plural y compartida" que "encierra al individuo en un aislamiento que hace que sea fácil caer en la lógica de la ideología, o más bien que la única facultad de la mente humana que no necesita que el ego, del otro o del mundo funcione, $y$ que es independiente tanto de la experiencia como de la reflexión". El individuo, por lo tanto, "se escapa de las responsabilidades del pensamiento $y$ del juicio autónomo", es decir, vive una fase de "atrofia de la facultad del juicio" y obedece "cadavéricamente las normas del contexto en el que vive, cualquiera que sea" (Pansera, 2017, p. 133). Los animal laborans habitan en una sociedad "donde la insatisfacción generalizada cede temporalmente $y$ engañosamente solo en la creciente sucesión de producción y consumo, y en la única y sola exaltación del dominio absoluto de los animal laborans" en sí misma y donde es cada vez más evidente la incapacidad de expresarse y pensar entendido como "pensar desde el punto de vista de otra persona" (Pansera, 2017, p. 135-137).

En este escenario en el que "la gente común tiene la impresión de ser cada 
vez más irrelevante", donde se teme que la elite económica del siglo XXI ya no necesite a la gente, la raza humana, aunque esté enojada y decepcionada "no abandonará la narrativa liberal porque no tiene alternativa" $y$ "eventualmente volverá sobre sus pasos". ¿Pero "tal vez los seres humanos no deberían dejar de lado la idea misma de una sola narrativa global" y asumir la tarea, la responsabilidad de "crear una narrativa actualizada para el mundo" ? (Harari, 2018 , p. $27-28$ y $35-37$ ).

Si la idea de que en el 2050 nos encontraremos ante una "clase inútil" nacida de la falta de trabajo o de una educación adecuada apocalíptica y especulativa, seguramente será necesario enfrentar "una resistencia mental insuficiente al cambio" (Harari, 2018, p. 64). De hecho, la automatización y el aprendizaje automático ya han modificado suficientemente el mercado laboral con consecuencias de empleo no despreciables y realizan cambios contínuos en casi todos los campos profesionales, imponiendo a la agenda pública una seria reflexión sobre nuevos puestos de trabajo y, en consecuencia, sobre el sistema educativo.

Hasta ahora, las máquinas han estado compitiendo con las personas solo desde el punto de vista físico, pero a partir de ahora se abre un escenario en el que el desafío también podría ocurrir a nivel cognitivo: "la inteligencia artificial hoy en día comienza a superar el rendimiento del Hombres en un número creciente de habilidades y tareas, incluida la comprensión de la dinámica emocional humana" también gracias a los descubrimientos en los campos biológico y social (Harari, 2018, p. 44). La continua investigación neurocientífica y en el campo de la economía del comportamiento permiten un conocimiento cada vez más refinado de los procesos de toma de decisiones, tanto que empezamos a hablar de "piratear seres humanos" (Harari, 2018, p. 44). El discurso sobre la era de la reproducibilidad digital se centra en el impacto que las tecnologías tendrán en el trabajo humano, pero descuida, casi por completo, las consecuencias que éstas producirán en el funcionamiento de la democracia. Además de las desigualdades económicas, "determinado por el extraordinario y rápido enriquecimiento de los "propietarios" de esas tecnologías, y en particular de aquellos que administran plataformas que llegan a un gran número de usuarios" como Amazon (Franzini, 2019), de hecho es necesario tener en cuenta el poder persuasivo y manipulador de las tecnologías digitales alimentadas por big data y su gratuidad.

A la larga, ninguna área de nuestra vida, ningún trabajo, será completamente inmune a la amenaza de la automatización: tendremos que estructurarnos para resistir los cambios frenéticos "tendremos que explorar nuevos modelos para la sociedad posttrabajo, para las economías posttrabajo y para las políticas post-empleo (...) El primer paso es admitir con honestidad que los modelos sociales, económicos y políticos que hemos heredado del pasado son inadecuados para enfrentar este desafío" (Harari, 2018, p. 67). 
En este escenario catastrófico, sin embargo, no debemos olvidar que "los seres humanos están dotados de cuerpos". A pesar de la crisis y la desintegración de las comunidades humanas, debido a la afirmación de comunidades virtuales que ofrecen sustitutos para las relaciones, generando vidas cada vez más solitarias en un mundo cada vez más interconectado, de hecho, "las comunidades físicas poseen una profundidad que no es comparable a aquellas de comunidades virtuales, al menos no en un futuro próximo". Por ejemplo, "si estoy enfermo en casa en Israel, mis amigos en línea de California pueden hablar conmigo, pero no pueden traerme un caldo caliente o una taza de té" (Harari, 2018, p. 133 y 138139). Por lo tanto, aunque la tecnología nos aleja de nuestros cuerpos, intentando sabotear las interacciones físicas directas, será nuestra corporeidad, nuestra tendencia intrínseca a relacionarnos, lo que nos salvará de la desilusión, del alejamiento, la alienación, la desorientación, pero también del desempleo masivo y la crisis de nuestro sistema educativo.

Las relaciones caracterizan nuestra condición humana, nuestra vida se construye en relaciones: "existimos dentro de una relación porque de una relación venimos", "somos seres relacionalmente insuperables" (Mortari, 2017, p. 96). Esta característica es confirmada por el desempeño del mercado laboral. Hay fases de la vida como la infancia, la enfermedad y la vejez (esta última, hoy, cada vez más prolongada, gracias al aumento de la esperanza de vida) en las que inevitablemente debemos confiarnos a los demás: "nuestra autonomía no es nunca un logro", la dependencia, aunque sea gradualmente diferente, es nuestra condición estructural (Mortari, 2017, p. 97). Por esta razón, sectores como la industria del cuidado personal seguirán siendo "un bastión humano durante mucho tiempo" (Harari, 2018, p. 52) y es a estos a los que el sistema educativo debe mirar cuidadosamente para poder saber cómo impartir lecciones útiles para las generaciones que tendrán que vivir y trabajar en el siglo XXI.

¿Qué entrenamiento, entonces, para un mercado laboral en el que ya no estamos seguros de nada? Mientras tanto, una "formación permanente como un nuevo derecho de ciudadanía": proclamarla como tal significa ponerla al centro de un nuevo pacto entre ciudadanos, empresas, escuelas, universidades, investigación y territorio, así como al interno de la actividad contractual ordinaria del sindicato y no tratarlo como una herramienta para ser utilizado en tiempos de crisis; significa establecer mecanismos para certificar competencias que van más allá de los juegos de las partes y encuentran su fundamento en la persona en todas las fases de su vida (Ranieri 2010, p. 132, en Gramolati y Mari, 2010). Con el fin de la estabilidad del lugar de trabajo y el empleo permanente, se solicitará capacitación continua para garantizar la empleabilidad en lugar de un puesto permanente. El objetivo será entrelazar el trabajo, la capacitación y las actividades de investigación capaces de 


\author{
"construir formas de organización del \\ trabajo capaces de aprender, responder \\ a los nuevos imperativos del \\ conocimiento $y$, por lo tanto, \\ convertirse en organizaciones que crean \\ conocimiento" (Trentin, \\ 2009),"comunidad de pares, \\ horizontalmente", "en la que las \\ personas tienden a agregarse \\ independientemente del sector" (Ranieri \\ 2010, p. 134, en Gramolati y Mari, \\ 2010).
}

Entonces, ¿qué escuela en una sociedad donde el cambio será la única certeza y en la que no sabemos qué habilidades se necesitarán para vivir y trabajar? Inevitablemente, tendremos que abandonar la "teoría educativa de la línea de ensamblaje" y comenzar a educarnos para "ser serenos en lo desconocido" para "aceptar lo desconocido", para "continuar aprendiendo a reinventarnos" a cualquier edad, a "considerar la extraña normalidad", volver a hacer que la antigua exhortación griega "conócete a ti mismo" para evitar que todas nuestras elecciones se confíen a la autoridad de los algoritmos (Harari, 2018, p. 388-391).

Sin embargo, como somos "seres relacionales", cuidarnos a nosotros mismos no es suficiente, "necesitamos lo que otros nos pueden dar", siendo "seres carentes de ser e íntimamente relacionales" tenemos una necesidad ineludible de "ser objetos de acciones de cuidado", tenemos que "aprender a cuidar a los demás". Cuidar debe entenderse como una práctica, como un proceso, como una forma de relacionarse con el otro y no simplemente como amar, apoyar,

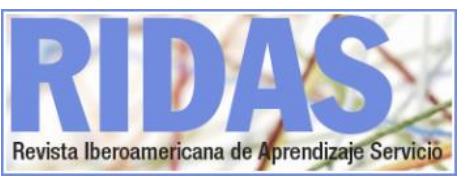

consolar, cuidar de los demás. Cuidar al otro es lo que da forma a nuestro ser, es decir, a nuestra vida, lo que nos hace encontrar nuestro lugar en el mundo, por lo tanto, lo interpretamos como un "servir" (Mortari, 2012, p. 65-66). La escuela del siglo XXI, por lo tanto, tiene la tarea de dedicarse al cuidado, de enseñar a prestar atencióni, concentrarse, escucharse a sí misma y a los demás. Cuidar consiste en "autocuidado" y "cuidado de los demás", pero los dos planos no son inconexos y también la intención que lo dirige es producir un doble beneficioii, hacia nosotros y hacia los otros. El cuidado es una "disposición al ser", que no es ser sino "ser capaz de llegar a ser" o "ser posible", pero no se puede poner en práctica por sí sola porque "estamos con los demás", en relación. Cuidar de uno mismo sirve para dar una mejor forma al propio ser, que puede florecer si, y solo si, también cuidamos a los demás: "el trabajo educativo", por lo tanto, "consiste en cuidar del otro para que el aprenda a cuidarte". La cura "sigue estando inmersa en el bien", que es "la base sólida de nuestra vida", que nos permite "vivir con placer" (Mortari, 2017, p. 94-99). En resumen, enseñar sobre cuidados también significa enseñar a vivir (Morin, 2014) con placer, en la relación, sentir empatía y compasión por los demás, "pensar con el corazón" (Mortari, 2017, p. 101) o "reunidos en las profundidades", entrando en contacto con el núcleo de nuestro ser y cuestionando "el yo en relación con el mundo que vive, las relaciones que vive y las situaciones que experimenta". En esta perspectiva, el aprendizaje debe entenderse como la 
práctica del yo, en relación con los demás, con el contexto social, como "un diálogo silencioso de la mente consigo mismo pero también con su lado social, intersubjetivo (...) dentro de una comunidad de práctica de autoaprendizaje" que puede tener "efectos transformadores" y, educar, como una promoción en el otro de la "capacidad de dar forma a uno mismo (autoformación)" y por lo tanto en "desarrollar la orientación ética de haber cuidado de la existencia y en el aprendizaje de las técnicas que nos permiten dar forma a nuestro propio ser" (Mortari, 2008, p. 48-49 y 54-55).

\section{Redescubrir el valor innovador y democrático de las competencias}

Desde muchos aspectos el sistema educativo está llamado a adaptarse a la nueva realidad y a los estímulos que son sobre todo externos. Al final del siglo pasado la gradual capacidad de medición de la economía ha fagocitado tambien el sistema educativo, en ocasiones asimismo prefiriendo la teoría cognitiva del aprendizaje sobre la teoría constructivista. El interés económico por el capital humano se determinó sobre todo en consideración a su indispensabilidad en frente a la introducción continua de innovaciones tecnológicas y organizativas de las cuales depende la productividad de los factores. En realidad, a partir de la década del setenta, para el crecimiento económico, el capital humano se trasformó en una inversión más importante que la del capital físico y que del progreso tecnológico, por este motivo el uso de indicadores de calidad educativa se ha extendido a escala internacional. Si antes el capital humano tenía solo una medida cuantitativa (nivel de escolaridad, gasto público en educación, etc), en las últimas décadas los instrumentos cualitativos han emergido, sobre todo, dirigidos a medir el desempeño de los estudiantes con exámenes estandarizados y el progreso escolar.

La afirmación de la economía del conocimiento, que ha identificado en el término competencia el posible vínculo entre el mundo de la educación y el mercado laboral, de hecho ha favorecido el marco cognitivista. La medición normalizada de las competencias como respuesta a la necesidad de una mayor conexión entre formación formal e informal ha facilitado una lectura funcionalista y de alguna manera mercantilista de ellas, resultante de la competición entre sistemas y entre individuos.

Sin embargo, asociado con la presión sobre el desempeño, el creciente individualismo nutre y se nutre del espíritu competitivo que potencia la singularidad independientemente de los demás y ante la explosión de situaciones gravemente problemáticas se sumerge en orgías de compasión y caridad (Bauman, 2003), perdiendo la fuerza de los vinculos sociales y del bienestar personal y colectivo. Además, siempre en las ultimas decadas, a nivel europeo e internacional, los efectos derivados del uso generalizado de las tecnologías de información, la presión del mercado global y la renovación científica y técnica acelerada se han convertido en retos verdaderos, tanto para el mundo del trabajo como para la educación. Especialmente en Italia, el crecimiento de la oferta de capital 
humano calificado no ha tenido una demanda correspondiente por parte de las empresas y de la administración pública, debido a la especialización productiva en sectores con tecnología madura, al tamaño pequeño de las empresas y a un modelo competitivo orientado a la reducción de costos en lugar de una inversión innovadora (Alessandrini, 2012).

En este clima de crisis continúa y creciente inseguridad, a pesar de la abundancia de estudios y encuestas, el sistema italiano de educacion escolar muestra signos cada vez más evidentes de una emergencia educativa: abandono escolar persistente, inequidad del sistemaiii, difusión de los fenómenos de bravuconería, incremento en los litigios en la relación entre la escuela y las familias, pérdida para el docente del rol social de referencia para la comunidad. Lamentablemente, con el debilitamiento de los sentimientos comunitarios está en riesgo la cultura de la democracia misma que, por el contrario, se basa en los principios de solidaridad, responsabilidad y compromiso. Si la educación es el centro neurálgico de cualquier organización social, hoy y gracias a la construcción progresiva de un psicópoder sufre de un sentimiento estructural de irresponsabilidad, de indiferenciación entre menores y adultos, de individualismo, apariencia, ruptura de vínculos, incapacidad para reconocer lo que nos une a los demás, narcisismo de consumo (Stiegler, 2014).

\section{Si a lo largo del siglo XX y}

especialmente en el último cuarto de siglo la pedagogía ha sido objeto de una fuerte confrontación política, buscando una revolución educativa y una liberación del autoritarismo de las instituciones, con el cambio de milenio el debate se ha debilitado considerablemente y las experiencias didácticas han progresado adaptándose a nuevas encuestas cualitativas en un sentido eficiente y al impulso tecnológico que las engloba. Después del fermento de los teóricos de la clase de laboratorio, de la escuela cooperativa, de la desescolarización; la posterior revolución de la informática y los medios de comunicación ha introducido nuevas formas de pensar y comunicar, aplastando los lugares tradicionales de socialización y sacando a relucir la idea dominante que todos deben actuar como individuos aislados y en competición con otros. De hecho se ha producido una convergencia entre las instancias de la pedagogía libertaria y de la pedagogía neoliberal, que ha colocado la libertad del individuo atomizado en el centro del proceso educativo. En este punto, ante la continua incapacidad de alcanzar objetivos económicos vinculados a la educación y la creciente emergencia educativa (Goussot, 2011), necesitamos retomar los principios y las ideas de ese fermento y referirnos a quien, con visión de futuro en la lucha contra las instituciones autoritarias, querían partir del conjunto de conexiones que forman la persona, de la red de relaciones sociales y culturales.

Ante la fijación en el desempeño individual, el concepto de aprendizaje en los últimos veinte años ha evolucionado considerablemente, 
estableciendo algunos principios fundamentales:

- el aprendizaje relevante implica una acción social y distribuida en el contexto (Hattie: 2009);

- la modificación cognitiva es el resultado de un proceso de interacción continua con personas, artefactos y problemas del contexto;

- la formación de categorías de compentencias que facilitan la inferencia está determinada por el contexto.

La evidencias de la investigación podrían, por lo tanto, ayudar a transformar los contextos de aprendizaje, con el fin de hacerlos más consistentes con los objetivos que se le da al sistema educativo (Ellerani, 2017). Por otra parte la afirmación que el conocimiento formal debe convertirse en competencias a partir de las primeras enseñanzas de Dewey ha tenido como propósito principal el de socavar la planta secular de acceso al conocimiento, de transmisiones de saberes, de las prácticas disciplinarias de la mente. Frente a una situación social mutable, con contínuos cambios tecnológicos, productivos y comerciales, la investigación educativa ha vinculado al paradigma de las competencias la crítica de las formas de aprendizaje tendientes al verbalismo y sin conciencia (Baldacci, 2006). Investigación educativa que, mirando los mecanismos de aprendizaje como una actividad esencial para el ser humano, ha reconocido las condiciones para el aprendizaje implícito en situaciones informales y la modulación del aprendizaje formal por parte de los informales que, a través de continuas solicitudes informativas y organizativas, desafía los sistemas estructurados de educación (Margiotta, 2016). Si el cerebro está hecho para aprender y frente a una dotación genética inicial, gracias a su arquitectura conectiva, el aprendizaje depende de la posibilidad de crear tantas conexiones neuronales como sea posible a través de las sinapsis, es la variedad de interacciones con el entorno natural y social lo que aumenta las competencias de cada uno. Entoces no es el individualismo, mas bien el fortalecimiento de las relaciones sociales, rendidas aún más significativas por la conexión con el conocimiento, lo que aumenta las competencias.

Si en la economía basada en el conocimiento, el capital humano de las competencias ayuda a hacer malabares en el juego de sillas, aprender la responsabilidad y solidaridad de la relación con el otro mantiene viva la democracia donde la enseñanza y la educación deben nutrirse mutuamente a través de la experiencia y la participación periférica legítima (Lave y Wenger, 2006). Efectivamente la atención a las habilidades transversales, ante el cambio constante que predice la distorsión del mercado laboral con la pérdida de empleos históricos y la aparición de ocupaciones inéditas, resalta la importancia del pensamiento crítico, de colaboracion con otros, de capacidad de autoevaluación y de responsabilidad relacional. En el desafío para la construcción de un sistema educativo para el siglo XXI, entre los factores que 
más influyen en el éxito escolar, surge el pasaje de la responsabilidad administrativa a la responsabilidad profesional de investigación educativa (Schleicher, 2018). De ahí el valor científico de la difusión de experiencias de aprendizaje-servicio, un enfoque educativo que permitiría en Italia redescubrir las afirmaciones de Raffaele Laporta (1975) sobre la importancia de la comunidad educadora.

\section{Redescubrir el valor de la comunidad educadora a través del aprendizaje-servicio}

El valor del aprendizaje no se limita a las habilidades de entrenamiento, funcional al mundo productivo, sino incluye en el desarrollo de la persona la plena adquisición del derecho de ciudadanía y sin duda el empuje de eficiencia orientada, que de hecho ha provocado la segregación del sistema escolar italianoiv, no facilita el logro de los objetivos establecidos para un crecimiento inteligente, inclusivo y sostenible, porque la falta de equidad en el sistema aclara las deficiencias de quienes tienen más dificultades y también limita el desarrollo de aquellos que tienen una ventaja (Schleicher, 2018). De la concentración en el único conocimiento nocional, más fácilmente medido de forma estandarizada, el debate económico sobre la educación también se ha enriquecido gracias al enfoque de la capacidad y a la afirmación de las habilidades de carácter, promoviendo de echo la adición de la tercera dimensión del aprendizajev ${ }^{v}$. Este valor más complejo del aprendizaje promueve una relación generativa que combina desarrollo y calidad de vida para el individuo, el cual crece en una relación contínua consigo mismo y con la sociedad participada que lo rodea (Stigliz, Sen y Fitoussi, 2009).

Dado el redescubrimiento del valor de las relaciones sociales y de la importancia del desarrollo humano de la persona que nutre y se nutre de la red de relaciones solidarias, para poder contrarrestar los efectos de la atomización y el individualismo precario, el aprendizaje-servicio emerge como un paradigma capaz de combinar el estudio en el marco formal de la institución educativa con el desarrollo del territorio, gracias a la posibilidad de asumir sus problemas. Promoviendo una idea de aprendizaje enriquecida por la profundidad de los vínculos de solidaridad intergeneracional, intercultural y ambiental. Fovoreciendo la retroalimentación continua "dentro" y "fuera" del aula, gracias a una reciprocidad que se convierte en un constructo teórico que permite comprender la relación de aprendizaje generativo, como una afirmación continua de la libertad.

Como recordó Hannah Arendt (1991), la escuela es la transición del mundo privado al público, donde los educadores representan para los jóvenes un mundo del que deben declararse responsables, con una responsabilidad que está implícita en la introducción a un mundo en constante cambio, en el que los jóvenes deben introducirse poco a poco. "La educación es el momento que decide si amamos al mundo lo suficiente como para asumir la responsabilidad por ello y salvarlo de la ruina, lo cual es inevitable sin renovación, sin la llegada de nuevos 
seres, de los jóvenes. En la educación también se decide si amamos a nuestros hijos para no expulsarlos de nuestro mundo dejándolos a merced de ellos mismos, tanto de no quitarles de sus manos la posibilidad de emprender algo nuevo, algo impredecible para nosotros; y prepárelos en cambio para la tarea de renovar un mundo que será común a todos." (Arendt, 1991, p. 255).

En la relacion educativa, recordó Arendt en esa lección, la autoridad del docente es un elemento clave de la responsabilidad y la crisis de la educación depende precisamente de la crisis de autoridad en todos los ámbitos de la vida pública y política, pero además, el hecho de que los adultos querían deshacerse de la autoridad significa que se niegan a responsabilizarse del mundo en el que introdujeron a sus hijos. Aunque expresándose en un período anterior a los grandes movimientos de protesta, ya ilustraba los peligros inherentes a la pérdida de autoridad que podría llevar a que todos asuman la responsabilidad como negación general.

El deseo de no huir a a la necesidad de una responsabilidad generalizada que evite ese tipo de autodestrucción inherente al individualismo atomista conduce a la promoción de la educación generalizada a nivel comunitario. No solo Freire, también Laporta (1996) y Lombardo Radice (1976) tomaron en consideración toda las conexiónes que forma la persona en la red de relaciones sociales y culturales condicionando su libertad. Así la promoción de la educación a la elección, de la costrucción de la autoconciencia y de la inteligencia relacional que puede llevar al individuo a desarrollarse, entretanto modificando cosas y personas a su alrededor, los llevó a dar gran importancia a la comunidad y al estudio como esfuerzo permanente de comprensión tratando de evitar el dogmatismo. Si Lombardo Radice (1976) se centró en la mediación de la experiencia colectiva que enmarcaba la socialización, Laporta asignó gran importancia a la responsabilidad mutua y al desarrollo de una sociedad democrática e inclusiva. "La educación está por lo tanto a garantizar que en la relación interpersonal toda persona esté protegida, la relación educativa es una relación típica entre desiguales que deben convertirse en iguales: por lo tanto debe ser una práctica constante de igualdad. Enseñar intelectualmente la igualdad a través de mensajes verbales es inútil! Necesitamos hacerlo en vivo." (Laporta, 1975, p. 7-8)

Comparado con esos años, hoy el predominio de lo virtual y la pérdida de muchas oportunidades para la socialización espontánea han frustrado muchas posibilidades de experiencia. Por eso un enfoque educativo que quiera facilitar el desarrollo humano, enriqueciendo a los jóvenes y personas en general con conocimientos motivados o libremente elegidos en relación a la comparación con el otro y el cuidado de la relación humana y ambiental, hace necesario reactivar los circuitos de participación democrática. En este marco el aprendizaje-servicio constituye una referencia pedagógica capaz de unir motivación y libertad para involucrar a los jóvenes en el desarrollo 
del conocimiento y de experiencias concretas que reactiven las relaciones sociales y democráticas. Así, gracias al aprendizaje-servicio, la comunidad se vuelve educadora y formativa de las competencias necesarias para la persona y el grupo, humanizando las estructuras gracias a la renovada habilidad de cada uno para cuidar y romper aislamientos y exclusiones; en una relación generativa de aprendizaje multidireccional -estudiante-estudiante, estudiante-docente, docente-docente, estudiante-personas externas etc - .

Este paradigma educativo permite apreciar el desarrollo humano a través de la elección individual y comunitaria. Permite superar la búsqueda de una receta estereotipada de una enseñanza seductora, que captando sin crítica la atención del estudiante restaura la asimetría de la autoridad e inhibe la liberación del espíritu crítico.

Simultáneamente permite capturar la ramificación de enlaces y su valor en el desarrollo del conocimiento, mucho más generativo que la árida transmisión de contenidos tecnológicos. Por lo tanto aparece como un alejamientovi ${ }^{\text {, capaz }}$ de hacer que todos comprendan la estructura del sentido de aprendizaje, para lo cual más que la cosa es importante como sucede: a través de una motivación personal, condicionada por el marco social de referencia, pero decisiva para el desarrollo. "[Sobre la libertad] ciencia y educación deben contar para tener la fuerza de proyectar en el futuro, un futuro sin límites, el destino de nuestra especie." (Laporta, 1996,p. XXIV)

\section{Educar sobre la ética de la responsabilidad}

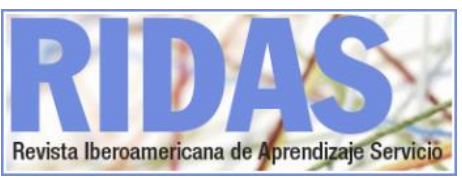

Cuidarse como un interés en el otro representa un remedio para el individualismo, para la existencia concebida de una manera aislada, atomística y competitiva: educar para cuidar significa, por tanto, educar para sentirse responsable, "tener que hacer algo" para otro, mirándolo y "considerándolo como una entidad valiosa que me mira" y "me desafía". Al actuar con cuidado, "vemos la realidad del otro como una posibilidad para nosotros" y, por lo tanto, actuamos "para eliminar lo que es intolerable, para reducir el sufrimiento y satisfacer una necesidad, para realizar un sueño". El término responsabilidad deriva del latín respondere: ser responsable significa, por lo tanto, no solo declarar, sino sobre todo dar una respuesta rápida, activa y cuidadosa que garantice el bienestar del otro, su sernosotros. Actuar con responsabilidad es actuar "con coraje porque creemos que no hay otra opción compatible con la necesidad de cuidar a los demás" (Mortari, 2008, p. 100-103).

En la sociedad postmoderna, en la que el individuo es "movido por un impulso ilimitado a la autorrealización, cerrado de manera entrópica en el circuito autorreferencial de sus deseos, que excluye toda alteridad, indiferente a la esfera pública y al bien común es incapaz de planificar" (Pulcini, 2009, p. 32), a partir de una escuela que educa a la ética del cuidado $y$, por lo tanto, de la responsabilidad, sería un gesto de buena política y devolvería valor a toda la comunidad educativa y, en particular, a los maestros, regresando para conferirles ese importante papel social perdido en la brecha entre pasado y 
futuro. En esta brecha entre el "ya no" y el "todavía no" (Arendt, 1991), el educador, está llamado a asumir una responsabilidad global, a crear un nuevo orden a partir de la escuela, a intentar recuperar preciosos fragmentos de tradición pedagógica, tratando de reconectarlos de una manera diferente. Preservar y conservar son de hecho una parte esencial de la actividad educativa que tiene la tarea de proteger siempre algo: "el niño del mundo, el mundo del niño, lo nuevo de lo viejo, lo viejo de lo nuevo (...). La educación debe ser conservadora precisamente por lo que es nuevo y revolucionario en cada niño: debe preservar la novedad e introducirla como algo nuevo en un mundo antiguo, que, sin importar cuanto pueda comportarse como un revolucionario, ante la generación" lo que ocurre siempre está desactualizado y cerca de la destrucción "(Arendt, 1991, p. 250-251).

¿Cómo pueden reconciliarse innovación y tradición en la escuela? ¿Cómo se educa la ética del cuidado y la responsabilidad? A través de un cambio de perspectiva que permite a los estudiantes asumir y desarrollar un pensamiento crítico que guía su acción en el mundo, por ejemplo, adoptando métodos de enseñanza como el aprendizaje-servicio que, comparándose con las necesidades reales del territorio, lleva a cabo un servicio comunitario, desarrolla un pensamiento reflexivo sobre la experiencia, educa a la responsabilidad a través de la relación (Mortari, 2017) $y$, por lo tanto, permite que se cambie una determinada cultura (Tapia, 2018). En el aprendizaje-servicio, el servicio en la comunidad, en el territorio, se convierte en una herramienta fundamental para el crecimiento personal que se combina con la enseñanza de competencias y se opone a los enfoques utilitaristas e individualistas.

Además el aprendizaje-servicio también representa una excelente forma de aprendizaje para maestros. Dewey nos recuerda que la investigación es un elemento esencial en los caminos de formación inicial de educadoras y educadores, porque permite una actitud más consciente hacia la propia práctica y permite el desarrollo de la autonomía y la responsabilidad. La formación en investigación se incluye en algunos documentos europeos recientes. En 2014, la Comisión Europea lo informa en Educación inicial para docentes en Europa: una visión general de las cuestiones políticas como una competencia fundamental junto a las habilidades reflexivas e interpersonales. En una escuela entendida como una comunidad de prácticas (Wenger, 1998), "o una comunidad capaz de dar voz a la experiencia y obtener conocimientos útiles para el desarrollo de la práctica docente", las habilidades reflexivas e interpersonales constituirán el núcleo de profesionalidad del docente. Desde el punto de vista del aprendizaje, hacer investigación significa escuchar problemas reales, a partir de experiencias, estar donde "ocurre la educación" (Mazzoni y Ubbiali, 2015, p. 245-250). Ninguna metodología de enseñanza, se centra en este objetivo como el aprendizajeservicio. Su institucionalización, en los circuitos académicos de la formación 
docente, permitiría el logro de importantes resultados hacia la plena realización de la comunidad educativa.

De hecho, el aprendizaje-servicio produce colaboración entre los miembros de la universidad y la comunidad en la cogeneración de conocimiento (investigación-acción), reciprocidad o beneficios para la comunidad y para los estudiantes y la consolidación de los lazos comunitarios entre la escuela y el territorio.

Un experimento que forma parte de esta tendencia es el proyecto Scuolalavoro e solidarietàvii, realizado en Toscana a partir del año escolar 2016/2017. La experiencia deriva de la necesidad de contrarrestar la ideología de la formación del capital humano, impuesta con una ferocidad cada vez mayor en el escenario escolar italiano de la última década y sancionada por la reforma escolar en el 2015, a través de la recuperación de la principios de la carta constitucional (en particular en los artículos 1-3-4) "que espera una escuela como una comunidad democrática que garantice el pleno crecimiento moral e intelectual de los ciudadanos trabajadores al perseguir su igualdad democrática en la diversidad individual". El proyecto, que llegó en el 2019 a su tercer año de vida, es el hijo del encuentro de dos metodologías didácticas que, aunque distintas, suelen utilizarse para concretar la relación entre las escuelas: por un lado, el aprendizaje-servicio "apunta a la educación para la ciudadanía activa y busca crear un vínculo entre el aprendizaje escolar y el servicio a la comunidad ", por otro lado, la alternancia entre la escuela y el trabajo, que en cambio "apunta al desarrollo de habilidades y busca conectar el aprendizaje al lugar de trabajo". (Betti y Lotti, 2018, p. 29-3031).

La naturaleza repentina y obligatoria de las rutas de alternancia de la escuela al trabajo impuesta por la Ley 107/2015 significó que la atención se centrara en la necesidad de cubrir el número de horas requeridas con actividades fuera del aula en acuerdo con las estructuras, profesionales escolares y empresas, que frecuentemente no se encuentran preparadas, en lugar de evaluar el impacto social y económico de las experiencias. La aplicación de los principios típicos del aprendizajeservicio a las vías de alternancia entre la escuela y el trabajo los ha llevado de vuelta a la via de la calidad en lugar de la cantidad. El proceso de investigaciónacción constituyó un momento de capacitación en acción para toda la comunidad educativa, y contribuyó de manera importante a su crecimiento y participación.

Ante la pérdida del valor social y cívico de la enseñanza-aprendizaje, cada vez es más necesario recurrir a prácticas que fortalezcan los vínculos y las colaboraciones entre las escuelas y el territorio, así como los vínculos internos con los institutos. Al tener el carácter de reciprocidad, el aprendizaje-servicio tiene un gran potencial en este sentido, ya que puede producir un doble cambio de visión: en los estudiantes, pero también en la comunidad, en la organización, en el territorio que interactúa con ellos. Entonces, ¿dónde empezar a desencadenar este círculo virtuoso? En primer lugar, capacitando, 
planificando y realizando cursos calificados y certificados sobre esta metodología de enseñanza, abiertos a todo el personal escolar en una perspectiva de aprendizaje-servicio doble. En segundo lugar, desde la creación de redes que involucren a escuelas, universidades, autoridades locales y asociaciones en línea con las coordinadas por CLAYSS en América Latina o REDAPS en España. En Italia, las experiencias de aprendizaje-servicio conscientes o inconscientes comienzan a ser muchas, pero el paso hacia la institucionalización de esta metodología sigue estando lejos y no puede separarse del fortalecimiento de las comunidades educativas y de la creación de un sistema de reflexión científica común que supervisa, evalua, mejora e implementa rutas existentes.

\section{Referencias bibliográficas}

Alessandrini, G. (2012). La pedagogia del lavoro. Education Sciences \& Society, 3(2).

Arendt, H. (1991). Tra passato e future. Milano: Garzanti.

Baldacci, M. (2006). Ripesare il curricolo. Roma: Carocci.

Bauman, Z. (2003). Una nuova condizione umana. Milano: Vita e Pensiero.

Betti, F., Lotti, P. (2018). Scuola-lavoro e solidarietà. Una direzione per

l'alternanza. Roma: Edizioni Conoscenza.
Ellerani, P. (2017). Costruire l'ambiente di apprendimento. Prospettive di cooperative learning, Aprendizaje y Servicio e problem-based learning. Roma: Lisciani scuola.

Gramolati, A. y Mari, G. (2010). Bruno Trentin Lavoro, libertà, conoscenza. Firenze: Firenze University Press.

Franzini, M. (2019). Tecnologie digitali, potere economico e democrazia. Roma: Menabò di Etica e Economia

Goussot, A. (2011). Pedagogie dell'uguaglianza. Saggi di pedagogia politica e filosofica. Roma: Edizioni del Rosone.

Harari, Y.N. (2018). 21 lezioni per il XXI secolo. Milano: Saggi Bombiani.

Laporta, R. (1975). La difficile scommessa. Firenze: La Nuova Italia.

Laporta, R. (1996). L'assoluto pedagogico. Saggio sulla libertà in educazione. Firenze: La Nuova Italia.

Lombardo Radice, L. (1976). Educazione e rivoluzione. Milano: Editori Riuniti.

Margiotta, U. (2016). Competenze e legittimazione nei processi formativi. Lecce: Pensa MultiMedia.

Mazzoni, V. y Ubbiali, M. (2015). Diventare insegnanti, tra ricerca e servizio. La pedagogia del Service Learning nella formazione dei futuri docenti. Form@re Open Journal per la formazione in rete, 3(15), 243-257.

Morin, E. (2014). Insegnare a vivere. 
Milano: Raffaello Cortina Editore.

Mortari, L. (2008). Conoscere se stessi per aver cura di sé. Studi sulla

formazione, 2, 45-48.

Mortari, L. (2012). La relazione di cura. Riflessioni sistemiche, 7.

Mortari, L. (2017). Educatori e lavoro di cura. Pedagogia Oggi, 2.

Mortari, L. (2017). La sapienza del cuore. Milano: Raffaello Cortina Editore.

Mortari L. (2017). Aprendizaje y Servicio. Per un apprendimento responsabile. Milano: Franco Angeli.

Pacchi, C. y Ranci C. (2017). White flight a Milano. La segregazione sociale ed etnica nelle scuole dell'obbligo. Milano: Franco Angeli.

Pansera, M. T. (2017). Hannah Arendt Per un'etica della responsabilità. Lezioni di teoria politica. Milano: Mimesis Edizioni.

Schleicher, A. (2018). World class. How to build a 21st-century school system. Parigi: OECD.

Stiegler, S. (2014). Prendre soin: de la jeunesse et des générations. Paris: Flammarion.

Trentin, B. (2009). Il lavoro e la conoscenza. Lectio doctoralis, Università Cà Foscari di Venezia.

i La atención es un acto intencional, un gesto ético, un trabajo que requiere poner el yo entre paréntesis para concentrarse en lo real. Su función es llevar un cierto fenómeno a la "presencia plena". Es un ejercicio en la base de cada actividad, es un acto cognitivo así como el presupuesto fundamental de tener cuidado. No prestar atención es como no vivir (Mortari, 2012, p. 68).

ii $\mathrm{Da}$ beneficium o "da algo al otro sin buscar nada del otro". Aquí encontramos la "calidad de atención donativa, su gratuidad (Mortari, 2008, p.101).

iii El sistema escolar en la Italia meridional e insular no solo parece menos efectivo en términos de resultados con respecto al de centro y especialmente con el del norte, pero también menos equitativo: la variabilidad de los resultados entre escuelas y entre clases en el primer ciclo de educación es consistente y en todo caso más alto en el norte y en el centro, de igual forma los porcentajes de alumnos con bajo estatus socioeconómico que no alcanzan niveles adecuados en las pruebas son más altos. (INVALSI, 2018)

iv Del estudio encargado por el Municipio de Milán a la Politécnica, basado en análisis cartográficos y estadísticas sobre la población de escuelas de primer ciclo en la ciudad y acompañado de información cualitativa y entrevistas realizadas en algunos barrios y municipios de la misma región, surge que el derecho obtenido en las últimas décadas por las familias de poder liberar el registro de niños de la relevancia de residencia ha favorecido la "huida de los blancos" ante situaciones de mayor incomodidad (Pacchi y Ranci, 2017). ${ }^{v}$ A la dimensión sincrónica del aprendizaje permanente (Ionglife) y al aprendizaje diacrónico en todos los lugares y ambientes de la vida cotidiana (lifewide), se agrega la dimensión circular del aprendizaje basado en la expansión de las raíces (lifedeep) gracias al compromiso conjunto de la comunidad y de cada uno por el gran desarrollo de las personas.

vi La referencia es a la técnica del alejamiento en el teatro épico de Brecht, para lo cual fue importante mostrar lo no dicho a través de la oración, para provocar 
una reacción con respecto a lo no dicho .

Lotti, P. y Betti, F. (2019). Aprendizaje-servicio al servicio de la Comunidad Educadora. RIDAS, Revista Iberoamericana de Aprendizaje Servicio, 7, 72-88. DOI10.1344/RIDAS2019.7.5 